OPEN ACCESS

Edited by:

Jun Li,

Lake Superior State University,

United States

Reviewed by:

Xinjiang $L u$,

Ningbo University, China

Jingqun Ao,

State Oceanic Administration, China

${ }^{*}$ Correspondence:

Qinghui Ai

qhai@ouc.edu.cn

Specialty section:

This article was submitted to

Comparative Immunology,

a section of the journal

Frontiers in Immunology

Received: 01 May 2021

Accepted: 25 May 2021

Published: 10 June 2021

Citation:

Wu M, Li Q, Mai K and Ai Q (2021)

Regulation of Free Fatty Acid

Receptor 4 on Inflammatory Gene

Induced by LPS in Large Yellow

Croaker (Larimichthys crocea).

Front. Immunol. 12:703914.

doi: 10.3389/fimmu.2021.703914

\section{Regulation of Free Fatty Acid Receptor 4 on Inflammatory Gene Induced by LPS in Large Yellow Croaker (Larimichthys crocea)}

\author{
Mengjiao $W u^{1}$, Qingfei $L i^{1}$, Kangsen $M a i^{1,2}$ and Qinghui $A i^{1,2 *}$ \\ ${ }^{1}$ Key Laboratory of Aquaculture Nutrition and Feed (Ministry of Agriculture and Rural Affairs), The Key Laboratory of \\ Mariculture (Ministry of Education), Ocean University of China, Qingdao, China, ${ }^{2}$ Laboratory for Marine Fisheries Science and \\ Food Production Processes, Qingdao National Laboratory for Marine Science and Technology, Qingdao, China
}

Free fatty acid receptor 4 (FFAR4) plays a key role in regulating the inflammatory response in mammals. The present study aimed to investigate the function of large yellow croaker FFAR4 on inflammation. In the present study, ffar4 was widely expressed in 10 tissues of large yellow croaker including gill, head kidney and spleen. Further studies showed that treatment of head kidney macrophages with agonists (TUG891 or GSK137647A) or overexpression offfar4 reduced the mRNA expression of pro-inflammatory genes induced by LPS, and increased the expression of ppar $\gamma$. Treatment of macrophages with antagonist AH7614 increased the mRNA expression of pro-inflammatory genes induced by LPS, and decreased the mRNA expression of ppar $\gamma$. In order to verify the immunomodulatory effect of PPAR $\gamma$, PPAR $\gamma$ was overexpressed in macrophages which significantly reduced the mRNA expression of pro-inflammatory genes il/6, il $1 \beta$, il/ , tnf $\alpha$ and cox2. Moreover, results of dual-luciferase assays showed that PPAR $\gamma$ downregulated the transcriptional activity of il 6 and il $1 \beta$ promoters. In conclusion, FFAR4 showed antiinflammatory effects on LPS-induced inflammation in large yellow croaker.

Keywords: large yellow croaker, FFAR4, inflammation, macrophage, PPAR $\gamma$

\section{INTRODUCTION}

Long-chain fatty acids, especially Omega- 3 fatty acids, have been shown to play an important role in inhibiting inflammatory processes (1). Over the past decade, researchers have found that Omega-3 fatty acids exert robust anti-inflammatory effects by activating $G$ protein-coupled receptors (2). FFAR4, also known as GPR120, is one of G protein-coupled receptors (3).

FFAR4 was initially identified as an orphan receptor in the human genome database in 2003 (4). FFAR4 is a lipid-sensing receptor which has seven transmembrane domains (3). FFAR4 is highly expressed in the macrophages, which played a crucial role in anti-inflammatory (5). Omega-3 fatty acids are natural ligands of FFAR4. Several Omega-3 fatty acids such as docosahexaenoic acid (DHA) and eicosapentaenoic acid (EPA) exert their anti-inflammatory effect through FFAR4 $(6,7)$. DHA treatment of RAW264.7 cells for $24 \mathrm{~h}$ inhibited the expression of COX-2 induced by LPS, and knockdown of FFAR4 eliminated the effect of DHA, indicating that FFAR4 mediated the anti-inflammatory effect of DHA by regulating the expression of COX2 (8). In addition, a recent study pointed that activation of FFAR4 in mice protected the liver from injury via PPAR $\gamma$ which is a 
transcription factor that regulates inflammation and insulin sensitivity (9). However, most of the research is limited to mammals and there is no research on anti-inflammatory mechanism of FFAR4 in fish.

Large yellow croaker (Larimichthys crocea) is an important economically fish in China (10). Meanwhile, large yellow croaker is a good research object for studying the mechanism of inflammation (11). The purpose of this study is to clarify the mechanism of fish FFAR4 on macrophage inflammation. These insights will help us develop nutritional strategies that target FFAR4 to alleviate fish inflammation and to facilitate the healthy development of large yellow croaker breeding industry.

\section{MATERIALS AND METHODS}

\section{RNA Extraction, and cDNA Synthesis}

All experiments conducted on fish followed the Management Rule of Laboratory Animals (Chinese Order No.676 of the State Council). Fish were anesthetized to minimize the pain before being dissected.

In the present study, tissues of large yellow croaker including gill, brain, eye, spleen, intestine, liver, muscle, head kidney, heart, and adipose tissues were collected for RNA extraction and RTqPCR. The entire RNA extraction process used TRIzol reagent (Takara, Japan). The total RNA extraction process was carried out according to the instructions. After adjusting the RNA concentration, Prime Script-RT reagent Kit (Takara, Japan) reverse transcription kit was used to reverse transcription of the extracted RNA to synthesize the first strand of cDNA.

\section{Real-Time Quantitative PCR (RT-qPCR)}

We used a real-time quantitative PCR instrument (Mastercycler ${ }^{\circledR}$ ep realplex, Eppendorf, German) to detect the expression levels of corresponding genes in large yellow croaker and analyzed the data. The primers used in this study were shown in Table 1. The $\beta$-actin gene was an internal reference gene. Before the experiment officially started, the designed primer sequence was verified for amplification efficiency. The amplification efficiency is approximately in the range of $0.9-1.1$, indicating that the primer can be used. Finally, we used the $2^{-\Delta \Delta C t}$ method to calculate the relative expression of each gene in large yellow croaker tissues and cells (12).

\section{Cell Culture, Treatment and Transfection}

Large yellow croaker head kidney macrophages were cultured in an incubator at $25^{\circ} \mathrm{C}$. The medium used for culturing macrophages of large yellow croaker is DMEM/F12. HEK293T cells were cultured in a $37^{\circ} \mathrm{C}$ incubator. The medium used for culturing HEK293T cells is DMEM with high glucose.

The FFAR4 agonists GSK137647A (MedChemExpress, USA), TUG891 (MedChemExpress, USA) and FFAR4 antagonist AH7614 (APE*BIO, USA) were dissolved with DMSO to a concentration of $50 \mu \mathrm{M}$, and then diluted in DMEM/F12 to a working concentration of $20 \mu \mathrm{M}$. Large yellow croaker macrophages were seeded in 12-well plates. After 24 hours of culture, LPS and the agonist (GSK137647A or TUG891) or antagonist AH7614 were co-incubated with macrophages for 12 hours, and then the cells were collected for RT-PCR. The working concentration of LPS was $50 \mu \mathrm{g} / \mathrm{mL}$.

The large yellow croaker FFAR4 was cloned into the pcDNA3.1EGFP vector (Invitrogen). The primers designed were shown in

TABLE 1 | Primer sequences for cloning and RT-PCR.

\begin{tabular}{|c|c|c|}
\hline Primer name & Sequence & Accession number \\
\hline FFAR4-RT-R & ACAAGGACCAGAAGAGTGCG & MW468049.1 \\
\hline IL-1 $\beta-R T-F$ & CATAGGGATGGGGACAACGA & XP_010734853.1 \\
\hline IL-1 $\beta-R T-R$ & AGGGGACGGACACAAGGGTA & XP_010734853.1 \\
\hline IL-6-RT-F & CGACACACCCACTATTTACAAC & XM_010734753.3 \\
\hline TNF $\alpha-R T-R$ & CCGTGTCCCACTCCATAGTT & EU931626.1 \\
\hline IFNh-RT-F & TCAGACCTCCGCACCATCA & KM501500.2 \\
\hline IFNh-RT-R & GCAACCATTGTAACGCCACTTA & KM501500.2 \\
\hline IL-8-RT-F & AATCTTCGTCGCCTCCATTGT & XM_010737667.3 \\
\hline IL-8-RT-R & GAGGGATGATCTCCACCTTCG & XM_010737667.3 \\
\hline IL-10-RT-F & AGTCGGTTACTTTCTGTGGTG & XM010738826 \\
\hline FFAR4-GFP-R & GCCACTGTGCTGGATATCTGCAGAAACCСCTGCCACAAGGTGCT & MW468049.1 \\
\hline PPAR $\gamma-p C S 2-F$ & CGATTCGAATTCAAGGCCTCTCGAG ATGCAAACACCAGGCAGAG & XM_010731330.2 \\
\hline PPAR $\gamma-\mathrm{pCS} 2-\mathrm{R}$ & CTCACTATAGTTCTAGAGGCTCGAGCTAATACAAGTCCTTATGA & XM_010731330.2 \\
\hline$\beta$-actin-RT-F & GACCTGACAGACTACCTCATG & GU584189 \\
\hline$\beta$-actin-RT-R & AGTTGAAGGTGGTCTCGTGGA & GU584189 \\
\hline
\end{tabular}


Table 1. The open reading frame of FFAR4 with deletion of the stop condon was inserted into pcDNA3.1-EGFP plasmid (Promega, USA) using ClonExpress II One Step Cloning Kit (Vazyme Biotech, China). Plasmid DNA was extracted using EasyPure HiPure Plasmid MiniPrep Kit (TransGen Biotech, China).

The genes were overexpressed in large yellow croaker macrophages through electrotransduction. Before electroporation, large yellow croaker macrophages were cultured in serum-containing medium without penicillin-streptomycin solution. $25 \mu \mathrm{l}$ OPTIMEM, $1 \times 10^{6}$ cells and $5 \mu \mathrm{g}$ of the pcDNA3.1-EGFP-FFAR4 plasmid which was to be overexpressed formed an electroporation system. Then, the mix was added to the electroporation cup for electroporation. After 24 hours of electroporation, the gene expression was observed under fluorescence microscope and the cells were subjected to LPS (Sigma, USA). After 12 hours of LPS incubation, the cells were lysed and RNA was extracted. Then, the mRNA expression of genes related to inflammation was detected.

\section{Plasmid Construction and Dual-Luciferase Reporter Assay}

The large yellow croaker ppar $\gamma$ was cloned into the pCS2 + vector (Invitrogen) (Table 1). The IL1 $\beta$ promoter plasmid (pGL6-IL1 $\beta$ ) and IL6 promoter plasmid (pGL6-IL6) were stored in our laboratory. The sequences of IL1 $\beta$ promoter and IL6 promoter were shown in Supplementary Figures $\mathbf{1}$ and 2, respectively.

In dual-luciferase reporter assay, the activity of firefly luciferase reporter gene (pGL6-IL1 $\beta$ and pGL6-IL6) was detected by using luciferin as substrate, and then the activity of renilla luciferasel reporter gene was detected by using coelenterazine as substrate when the firefly luciferase was quenched. The ratio of the two luciferases was the reporter promoter activity. Before the luciferase assay, HEK293T cells were seeded into 24-well plates. We used EZ Trans Plus cell transfection reagent (LIFE iLAB BIO, China) to transfect the reporter plasmids (pGL6-IL1 $\beta$, pGL6-IL6), transcription factor plasmids (pCS2-PPAR $\gamma$ ), and pRT-TK renilla luciferase plasmids into HEK293T cells. The activity of the firefly luciferase reporter gene and the renilla luciferase reporter gene were measured on the microplate reader using TransDetect ${ }^{\circledR}$ Double-Luciferase Reporter Assay Kit (Transgene Biotech, China). The ratio of the two readings was the fluorescence value after correcting the number of cells. The experiment was repeated four times.

\section{Statistical Analysis}

The data analysis software used in this experiment was SPSS 18.0. Independent $t$-tests and the Tukey's test were used on the data. All data were presented as mean \pm SEM (standard error of the mean). When $P<0.05$, the difference was statistically significant.

\section{RESULTS}

\section{Tissue Distribution of Large Yellow Croaker ffar4}

The mRNA expression of ffar4 was determined in different tissues of large yellow croaker, such as the gill, brain, eye, muscle, head kidney, adipose, liver, heart, intestine and spleen tissues (Figure 1).

\section{TUG891 or GSK137647A-Mediated Activation of FFAR4 Suppressed the LPS-Induced Expression of Pro-Inflammatory Genes}

In order to study the role of FFAR4 in the regulation of LPSinduced inflammation, in vitro studies were conducted in macrophages of large yellow croaker. LPS significantly increased the mRNA expression of ill $\beta$, il6, ils, tnf $\alpha$ and cox2, and significantly decreased the mRNA expression of ppar $\gamma$ in macrophages $(P<0.05)$ (Figure 2). TUG891 or GSK137647A treatment significantly decreased LPS-induced expression of $i l 1 \beta$, il6, ils, tnf $\alpha$ and $\operatorname{cox} 2(P<0.05)$ (Figure 2). TUG891 or GSK137647A significantly upregulated the mRNA expression of ppar $\gamma$ relative to the LPS treatment $(P<0.001)$ (Figure 2).

\section{AH7614-Mediated Inhibition of FFAR4 Increased LPS-Induced Pro-Inflammatory Cytokine Expression and Decreased the Expression of ppary}

LPS significantly increased the mRNA expression of $i l 1 \beta$, $i l 6, i l 8$, tnf $\alpha$ and $\operatorname{cox} 2$, and significantly decreased the mRNA expression of ppary in macrophages $(P<0.05)$ (Figure 3). AH7614 significantly up-regulated the mRNA expression of $i l 1 \beta, i l 6, i l 8$, tnf $\alpha$ and $\operatorname{cox} 2$, and significantly down-regulated the mRNA expression of ppary relative to the LPS treatment $(P<0.001)$ (Figure 3).

\section{Overexpression of ffar4 Suppressed LPS-Induced Pro-Inflammatory Cytokine Expression and Improved the Expression of ppary}

FFAR4 and GFP were overexpressed and the overexpression efficiency of FFAR4 and GFP was same under fluorescence microscope (Supplementary Figure 3). Following exposure to LPS, the mRNA expressions of ill $\beta$, il6, ils, cox 2 and $t n f \alpha$ increased significantly relative to the control group $(P<0.05)$ (Figure 4). However, LPS treatment significantly decreased the mRNA expressions offfar 4 compared with the control group $(P<$ 0.05) (Figure 4). Overexpression of ffar4 significantly improved the expression of ppar $\gamma$ and ifnh, and significantly decreased the expressions of $i l 1 \beta$, il6, il8, cox 2 and $\operatorname{tn} f \alpha$ compared to the LPS group $(P<0.05)$ (Figure 4$)$.

\section{Overexpression of ppar $\gamma$ Suppressed LPS-Induced Pro-Inflammatory Cytokine Expression}

Results above showed that ppary expression of the LPS group was significantly lower than the control group, and was significantly increased by the overexpression of ffar4. To investigate whether the expression of ppar $\gamma$ is related to inflammatory response in large yellow croaker, the macrophages was treated with LPS and ppar $\gamma$ was overexpressed. Consistent with the previous results, LPS significantly increased the mRNA expressions of $i l 1 \beta, \operatorname{cox} 2, \mathrm{il} 6$, ils and $\operatorname{tnf} \alpha$ relative to the 


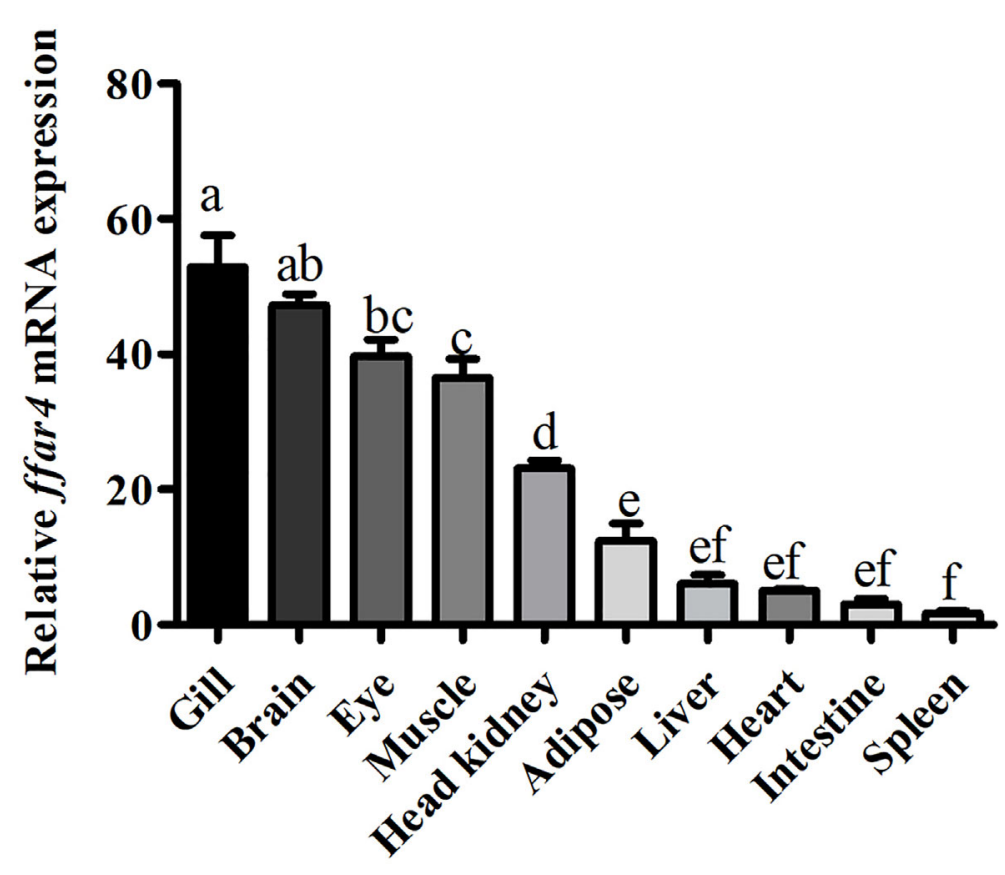

FIGURE 1 | Tissue expression of ffar4 in large yellow croaker. Data were shown as mean \pm S.E.M. ( $n=4)$. The same superscript letter means not significantly different, $P>0.05$. Values in bars bearing the same letters were not significantly different $(P>0.05)$.

control group $(P<0.05)$ (Figure 5). Overexpression of ppar $\gamma$ significantly decreased the expressions of $i l 1 \beta, \operatorname{cox} 2$, il6, il 8 and tnf $\alpha$ compared to the LPS group $(P<0.05)$ (Figure 5).

\section{The Regulatory Effect of PPAR $\gamma$ on the IL1 $\beta$ or IL 6 Promoter}

To determine the role of PPAR $\gamma$ in regulating transcriptional activity of pro-inflammatory genes, HEK293T cells were cotransfected with the IL1 $\beta$ or IL6 promoter luciferase reporter plasmid and the PPAR $\gamma$ expression plasmid, using PGL6-Basic and PCS2+ as controls in the dual-luciferase reporter assay. The transcription factor PPAR $\gamma$ significantly down-regulated the promoter activity of IL1 $\beta(P<0.01)$ and IL6 $(P<0.05)$ (Figure 6).

\section{DISCUSSION}

As a receptor for Omega-3 fatty acids, FFAR4 has many important functions in humans and other mammals. Many studies have shown that FFAR4 plays an important role in the treatment of diabetes (13), obesity (14), liver damage (9) and other immune diseases (15). However, there is almost no research on fish FFAR4.

In the present study, ffar4 was widely expressed in 10 tissues of large yellow croaker, which is consistent with mammalian (5). In mammals, the main function of FFAR4 is anti-inflammatory in immune organs and cells (16). Gill, head kidney and spleen are important immune organs of fish. Therefore, the expression of FFAR4 in gill, head kidney and spleen of large yellow croaker suggests that FFAR4 may be involved in regulating inflammation in

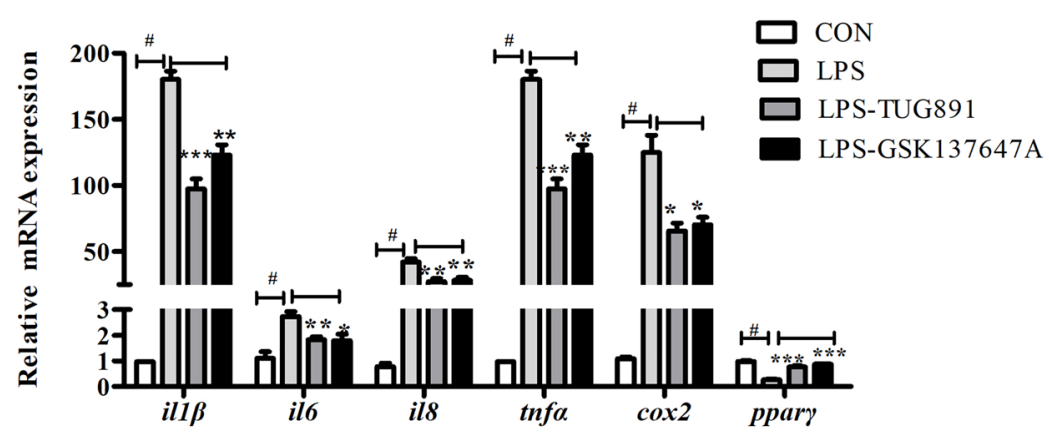

FIGURE 2 | Effects of FFAR4 agonist TUG891 or GSK137647A on the expression of LPS-induced il1 $\beta$, il6, il/, thf $\alpha$, cox2 and ppar $\gamma$ in macrophages of large yellow croaker. Data were shown as mean \pm S.E.M. $(n=4) .{ }^{\#} P<0.05,{ }^{\star} P<0.05,{ }^{\star \star} P<0.01,{ }^{\star \star \star} P<0.001$. 


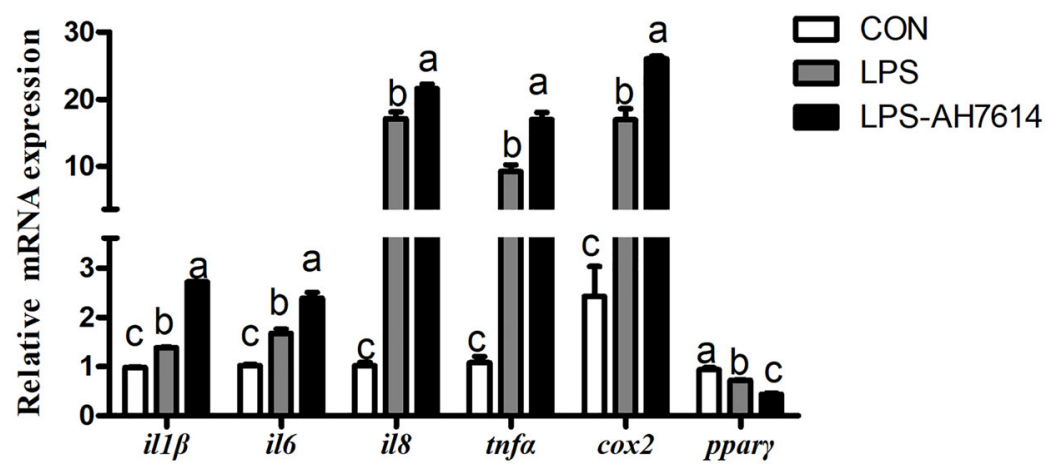

FIGURE 3 | Effects of FFAR4 antagonist AH7614 on the expression of inflammation related genes induced by LPS in macrophages of large yellow croaker. Data were shown as mean \pm S.E.M. $(n=4)$. For each gene, values in bars bearing the same letters were not significantly different among treatments $(P>0.05)$.

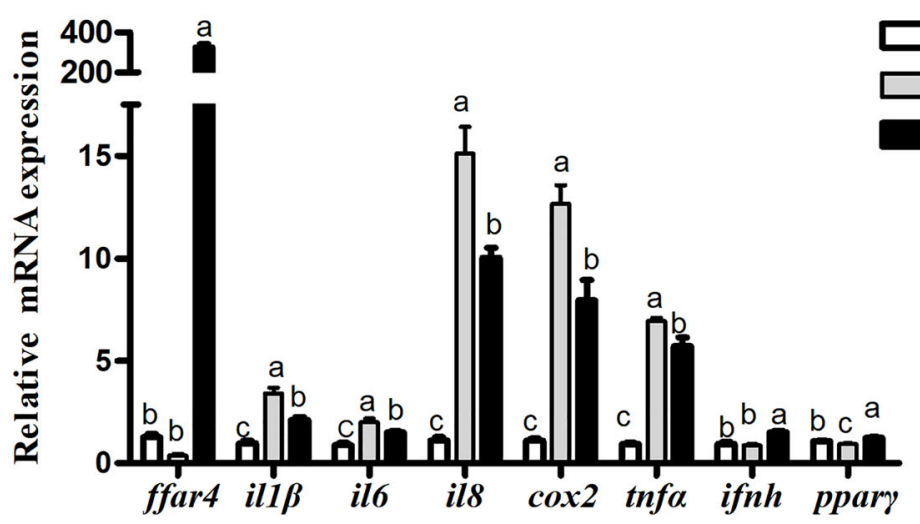

FIGURE 4 | Effects of FFAR4 overexpression on LPS-induced expression of il1 $\beta$, il6, il/, cox2, thf $\alpha$, ifnh and ppary in macrophages of large yellow croakers. Values were shown as mean \pm S.E.M. $(n=4)$. For each gene, values in bars bearing the same letters were not significantly different among treatments $(P>0.05)$.

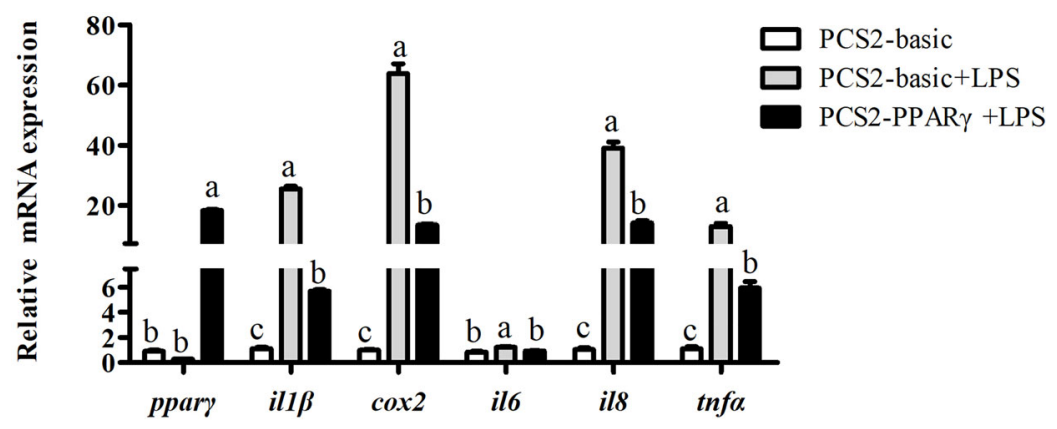

FIGURE 5 | Effects of PPARy overexpression on LPS-induced expression of il $\beta$, cox2, ill, il 8 , and thf $\alpha$ in macrophages of large yellow croakers. Values are presented as mean \pm S.E.M. $(n=4)$. For each gene, values in bars bearing the same letters were not significantly different among treatments $(P>0.05)$.

large yellow croaker. Among them, gill is not only immune organ of fish, but also the taste-sensing organ with the distribution of taste bud cells $(17,18)$. Studies in mammals have found that FFAR4 plays a taste perception role in taste bud cells $(19,20)$, so the high expression of FFAR4 in gill may also be related to taste perception. Compared with gills, FFAR4 has lower expression in head kidney and spleen. However, the function of the FFAR4 is not only affected by quantity, but also by activity. After being activated by the ligand, 


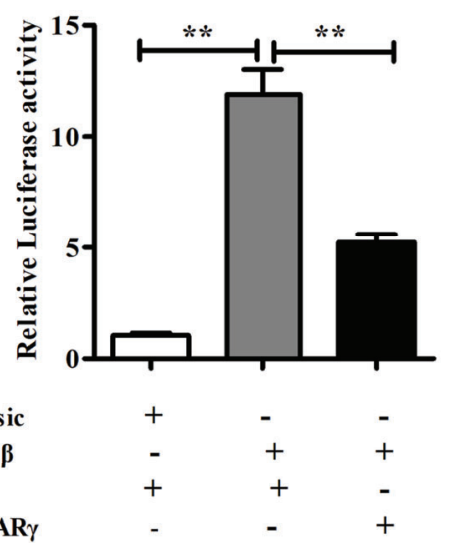

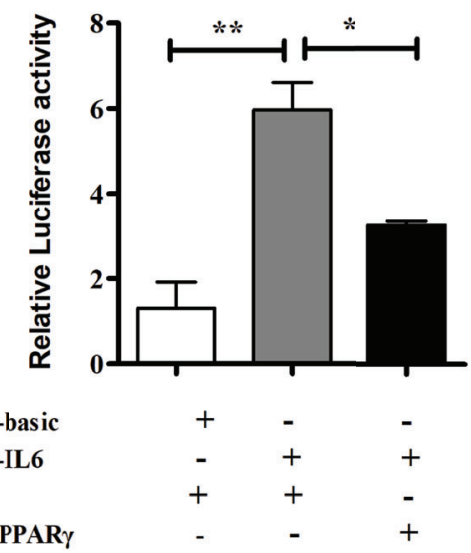

FIGURE 6 | Dual-luciferase analysis of overexpression of PPAR $\gamma$ on large yellow croaker IL1 $\beta$ (A) and IL6 (B) promoter activity in HEK293T cells. PRL-CMV and pGL6-Basic used as control. Data are presented as means \pm S.E.M. $(n=4) .{ }^{\star} P<0.05,{ }^{\star \star} P<0.01$.

FFAR4 expands the signal transmission step by step by regulating the generation of the second messenger, thereby completing the cell response (21). Thus, FFAR4 can exert a powerful immune effect even in small amounts. Head kidney is a unique immune organ of fish and plays a vital role in preventing the invasion of pathogens and maintaining the health of the body in large yellow croaker (22). So next we studied the function of FFAR4 in inflammation in head kidney macrophages of large yellow croaker.

Researches show that FFAR4 played an important role in regulating inflammation in macrophages of mammals $(23,24)$. In order to explore whether FFAR4 is involved in inflammatory regulation in large yellow croaker head kidney macrophages, we used LPS to induce an inflammation model of large yellow croaker macrophages. In this experiment, the co-incubation of FFAR4 agonist TUG891 or GSK137647A with LPS effectively alleviated the LPS-induced inflammatory response of large yellow croaker macrophages, and significantly reduced the expression of proinflammatory genes $i l 1 \beta$, il6, ils, cox 2 , and $t n f \alpha$. In addition, overexpression of ffar4 in large yellow croaker macrophages also inhibited inflammatory response induced by LPS. In contrast, the treatment of antagonist AH7614 aggravated the LPS-induced inflammatory response. Our results are consistent with previous findings $(8,9)$. Recently, some researches showed that FFAR4 can induce a significant decrease in pro-inflammatory cytokines, such as il1 $\beta$ (16), il6 (25), $\operatorname{tnf} \alpha$ (26) and cox-2 (8). Among them, COX-2 is a prostaglandin synthesis enzyme which has been proved that the increase of COX-2 contributed to the inflammatory response (27). COX-2 derived prostaglandins are involved in a variety of inflammatory diseases (28). Therefore, the result of the downregulation of $\operatorname{cox} 2$ and other pro-inflammatory genes by FFAR4 indicates that FFAR4 is involved in the inhibition of inflammation. In addition, FFAR4 overexpression also significantly increased the expression of ifnh, which has not been reported in mammals. IFNh is commonly regarded as important inflammatory cytokines involved in immune responses. Studies of mammals and fish reported that IFNh pathways was triggered in the inflammatory state to protect the host (29-31). Therefore, the increase of ifnh after FFAR4 overexpression may be related to the regulation of immune response.

Furthermore, an interesting result we observed was that both in the FFAR4 agonist incubation experiment or the ffar4 overexpression experiment, the expression of ppar $\gamma$ increased compared to the control group. Recently, many studies have confirmed the connection between FFAR4 and PPAR $\gamma$ (32). In 3T3-L1 adipocytes, FFAR4 promoted expression of ppar $\gamma$ through the $\mathrm{Ca}^{2+}$ and ERK1/2 signaling pathways to promote adipogenesis (33). In the Gillian's study, fish oil prevented liver injury by normalizing the expression of ppary in a FFAR4dependent manner (9). PPAR $\gamma$ has been well studied in mammals for its role in immune regulation $(34,35)$, but there is no related report in large yellow croaker. Therefore, in order to verify that the expression of ppary also exerts antiinflammatory effects in large yellow croaker macrophages, we studied the effects of overexpression of ppary on the LPSinduced inflammation of large yellow croaker. The results showed that overexpression of ppar $\gamma$ inhibited the expression of inflammatory genes induced by LPS, indicating that PPAR $\gamma$ indeed played a role in regulating immunity in large yellow croaker macrophages. PPAR $\gamma$ is a transcription factor that has been shown to regulate the expression of many inflammatory genes $(36,37)$. Thus, we speculated that large yellow croaker PPAR $\gamma$ regulates the expression of inflammatory genes by affecting promoter activity. As the largest group of cytokines, interleukins has an important function on host immune response (38). Interleukin-1 beta (IL1 $\beta$ ) and interleukin-6 (IL6) are important and representative interleukins which play crucial role in immune response $(39,40)$. Inflammation is often accompanied by an increase in IL1 $\beta$ and IL6 expression in large yellow croaker (41). However, the transcriptional regulation of PPAR $\gamma$ on IL1 $\beta$ and IL6 has not been reported in large yellow 
croaker. So dual-luciferase assays were carried out to study the effect of PPAR $\gamma$ on IL1 $\beta$ and IL6 promoter activity. The results of dual-luciferase assay proved that PPAR $\gamma$ can inhibit the activity of IL1 $\beta$ and IL6 promoters. In short, these results suggest that PPAR $\gamma$ may inhibit inflammation by suppressing IL1 $\beta$ and IL6 transcriptional activity.

\section{CONCLUSION}

In conclusion, the immunomodulatory function of FFAR4 is conserved in large yellow croaker macrophages. Activation or overexpression of ffar 4 can reduce the mRNA expression of proinflammatory genes induced by LPS and increase the mRNA expression of ppar $\gamma$ which inhibits the transcriptional activity of pro-inflammatory genes. Inhibition of FFAR4 aggravated the LPS-induced inflammatory response and decreased the mRNA expression of ppar $\gamma$. Regarding the specific signal pathways which FFAR4 regulates and how to regulate inflammation through $\operatorname{PPAR} \gamma$, further experiments are needed. Our results provide new ideas and strategies for alleviating the inflammatory response of large yellow croaker, and enrich the basic research on nutritional immunology of marine fish FFAR4.

\section{DATA AVAILABILITY STATEMENT}

The original contributions presented in the study are included in the article/Supplementary Material. Further inquiries can be directed to the corresponding author.

\section{REFERENCES}

1. Yang B, Zhou Y, Wu M, Li X, Mai K, Ai Q. Omega-6 Polyunsaturated Fatty Acids (Linoleic Acid) Activate Both Autophagy and Antioxidation in a Synergistic Feedback Loop Via TOR-Dependent and TOR-independent Signaling Pathways. Cell Death Dis (2020) 11:607. doi: 10.1038/s41419-02002750-0

2. Alvarez-Curto E, Milligan G. Metabolism Meets Immunity: The Role of Free Fatty Acid Receptors in the Immune System. Biochem Pharmacol (2016) 114:3-13. doi: 10.1016/j.bcp.2016.03.017

3. Fredriksson R, Hoglund PJ, Gloriam DEI, Lagerstrom MC, Schioth HB. Seven Evolutionarily Conserved Human Rhodopsin G Protein-Coupled Receptors Lacking Close Relatives. FEBS Lett (2003) 554:381-88. doi: 10.1016/s00145793(03)01196-7

4. Im DS. Orphan G Protein-Coupled Receptors and Beyond. JPN J Pharmacol (2002) 90:101-06. doi: 10.1254/jjp.90.101

5. Im D-S. Functions of Omega-3 Fatty Acids and FFA4 (GPR120) in Macrophages. Eur J Pharmacol (2016) 785:36-43. doi: 10.1016/j.ejphar. 2015.03.094

6. Yamada H, Umemoto T, Kakei M, Momomura S-I, Kawakami M, Ishikawa SE, et al. Eicosapentaenoic Acid Shows Anti-Inflammatory Effect Via GPR120 in 3T3-L1 Adipocytes and Attenuates Adipose Tissue Inflammation in DietInduced Obese Mice. Nutr Metab (Lond) (2017) 14:33. doi: 10.1186/s12986017-0188-0

7. Kang S, Huang J, Lee B-K, Jung Y-S, Im E, Koh J-M, et al. Omega-3 Polyunsaturated Fatty Acids Protect Human Hepatoma Cells From Developing Steatosis Through FFA4 (Gpr120). Biochim Et Biophys Acta Mol Cell Biol Lipids (2018) 1863:105-16. doi: 10.1016/j.bbalip.2017.11.002

\section{ETHICS STATEMENT}

The animal study was reviewed and approved by the Committee on the Ethics of Animal Experiments of Ocean University of China.

\section{AUTHOR CONTRIBUTIONS}

QA and MW designed the experiment. MW conducted the research. QL cloned FFAR4 gene, and constructed the plasmids. MW analyzed the data. MW wrote the manuscript. $\mathrm{KM}$ and QA revised the article. All authors contributed to the article and approved the submitted version.

\section{FUNDING}

This study was funded by the Key Program of National Natural Science Foundation of China (grant number 31830103); the National Science Fund for Distinguished Young Scholars of China (grant number 31525024).

\section{ACKNOWLEDGMENTS}

We thank KC and MY for their help during the research.

\section{SUPPLEMENTARY MATERIAL}

The Supplementary Material for this article can be found online at: https://www.frontiersin.org/articles/10.3389/fimmu.2021. 703914/full\#supplementary-material

8. Li X, Yu Y, Funk CD. Cyclooxygenase-2 Induction in Macrophages is Modulated by Docosahexaenoic Acid Via Interactions With Free Fatty Acid Receptor 4 (FFA4). FASEB J (2013) 27:4987-97. doi: 10.1096/fj.13-235333

9. Fell GL, Cho BS, Dao DT, Anez-Bustillos L, Baker MA, Nandivada P, et al. Fish Oil Protects the Liver From Parenteral Nutrition-Induced Injury Via GPR120-Mediated PPAR Gamma Signaling. Prostaglandins Leukot Essent Fatty Acids (2019) 143:8-14. doi: 10.1016/j.plefa.2019.02.003

10. Yao C, Huang W, Liu Y, Yin Z, Xu N, He Y, et al. Effects of Dietary Silymarin (SM) Supplementation on Growth Performance, Digestive Enzyme Activities, Antioxidant Capacity and Lipid Metabolism Gene Expression in Large Yellow Croaker (Larimichthys Crocea) Larvae. Aquacult Nutr (2020) 26:2225-34. doi: 10.1111/anu.13159

11. Yang B, Yin Z, Gao S, Chen M, Xu D, Mai K, et al. Characterization of Caspase8 and Its Role in the Regulation of Apoptosis-Related Genes in Large Yellow Croaker (Larimichthys Crocea). Aquaculture (2021). doi: 10.1177/ 1747493018778713

12. Livak KJ, Schmittgen TD. Analysis of Relative Gene Expression Data Using Real-Time Quantitative PCR and the 2(T)(-Delta Delta C) Method. Methods (2001) 25:402-08. doi: 10.1006/meth.2001.1262

13. Zhang D, Leung PS. Potential Roles of GPR120 and Its Agonists in the Management of Diabetes. Drug Design Dev Ther (2014) 8:1013-27. doi: 10.2147/dddt.s53892

14. Ichimura A, Hirasawa A, Poulain-Godefroy O, Bonnefond A, Hara T, Yengo L, et al. Dysfunction of Lipid Sensor GPR120 Leads to Obesity in Both Mouse and Human. Nature (2012) 483:350-U149. doi: 10.1038/nature10798

15. Milligan G, Alvarez-Curto E, Hudson BD, Prihandoko R, Tobin AB. Ffa4/ Gpr120: Pharmacology and Therapeutic Opportunities. Trends Pharmacol Sci (2017) 38:809-21. doi: 10.1016/j.tips.2017.06.006 
16. Oh DY, Talukdar S, Bae EJ, Imamura T, Morinaga H, Fan W, et al. Gpr120 Is an Omega-3 Fatty Acid Receptor Mediating Potent Anti-Inflammatory and InsulinSensitizing Effects. Cell (2010) 142:687-98. doi: 10.1016/j.cell.2010.07.041

17. Kinnamon JC, Royer SM. Synaptic Organization of Vertebrate Taste Buds. Proc Annu Meet Electron Microsc Soc Am (1994) 52:144-45. doi: 10.1017/ S0424820100168451

18. Hao Y-Y, Zhao J-L, Zhang R-Q, Chen X-W, Cao X-Y. Histological Characteristics of Taste Bud During Early Development of Mandarin Fish Siniperca Chuatsi. Chin J Zool (2018) 53:752-59. doi: 10.13859/j.cjz. 201805010

19. Matsumura S, Eguchi A, Mizushige T, Kitabayashi N, Tsuzuki S, Inoue K, et al. Colocalization of GPR120 With Phospholipase-C Beta 2 and AlphaGustducin in the Taste Bud Cells in Mice. Neurosci Lett (2009) 450:186-90. doi: 10.1016/j.neulet.2008.11.056

20. Matsumura S, Mizushige T, Yoneda T, Iwanaga T, Tsuzuki S, Inoue K, et al. GPR Expression in the Rat Taste Bud Relating to Fatty Acid Sensing. Biomed Res Tokyo (2007) 28:49-55. doi: 10.2220/biomedres.28.49

21. Moniri NH. Free-Fatty Acid Receptor-4 (GPR120): Cellular and Molecular Function and Its Role in Metabolic Disorders. Biochem Pharmacol (2016) 110:1-15. doi: 10.1016/j.bcp.2016.01.021

22. Li Q, Ai Q, Mai K, Xu W, Zheng Y. A Comparative Study: In Vitro Effects of EPA and DHA on Immune Functions of Head-Kidney Macrophages Isolated From Large Yellow Croaker (Larmichthys Crocea). Fish Shellfish Immunol (2013) 35:933-40. doi: 10.1016/j.fsi.2013.07.004

23. Yan Y, Jiang W, Spinetti T, Tardivel A, Castillo R, Bourquin C, et al. Omega-3 Fatty Acids Prevent Inflammation and Metabolic Disorder Through Inhibition of NLRP3 Inflammasome Activation. Immunity (2013) 38:115463. doi: 10.1016/j.immuni.2013.05.015

24. Cheshmehkani A, Moniri NH. Effects of Phosphodefective FFAR4 CTerminal Mutants on COX-2 Expression in Macrophages. FASEB J (2017) 31.

25. Tomita Y, Cakir B, Liu C-H, Fu Z, Huang S, Cho SS, et al. Free Fatty Acid Receptor 4 Activation Protects Against Choroidal Neovascularization in Mice. Angiogenesis (2020) 23:385-94. doi: 10.1007/s10456-020-09717-X

26. Wang L, Ren X, Tian X-F, Cheng X-L, Zhao Y-Y, Li Q-Y, et al. Protective Effects of GPR120 Agonist-Programmed Macrophages on Renal Interstitial Fibrosis in Unilateral Ureteral Obstruction (UUO) Rats. Biomed Pharmacother (2019) 117:109172. doi: 10.1016/j.biopha.2019.109172

27. Yu J, Ip E, Dela Pena A, Hou JY, Sesha J, Pera N, et al. COX-2 Induction in Mice With Experimental Nutritional Steatohepatitis: Role as ProInflammatory Mediator. Hepatology (2006) 43:826-36. doi: 10.1002/ hep. 21108

28. Dubois RN, Abramson SB, Crofford L, Gupta RA, Simon LS, Van De Putte LBA, et al. Cyclooxygenase in Biology and Disease. FASEB J (1998) 12:106373. doi: 10.1096/fasebj.12.12.1063

29. Oshiumi H, Matsumoto M, Funami K, Akazawa T, Seya T. Ticam-1, an Adaptor Molecule That Participates in Toll-Like Receptor 3-Mediated Interferon-Beta Induction. Nat Immunol (2003) 4:161-67. doi: 10.1038/ni886

30. Matsuo A, Oshiumi H, Tsujita T, Mitani H, Kasai H, Yoshimizu M, et al. Teleost TLR22 Recognizes RNA Duplex to Induce IFN and Protect Cells From Birnaviruses. J Immunol (2008) 181:3474-85. doi: 10.4049/jimmunol.181.5.3474
31. Zhu S, Xiang X, Xu X, Gao S, Mai K, Ai Q. TIR Domain-Containing AdaptorInducing Interferon-Beta (TRIF) Participates in Antiviral Immune Responses and Hepatic Lipogenesis of Large Yellow Croaker (Larimichthys Crocea). Front Immunol (2019) 10:2506. doi: 10.3389/fimmu.2019.02506

32. Paschoal VA, Oh DY. Revisiting PPAR Gamma as a New Friend of GPR120 in the Treatment of Metabolic Disorders. Adipocyte (2020) 9:649-52. doi: 10.1080/21623945.2020.1838186

33. Bae I-S, Park PJ, Lee JH, Cho E-G, Lee TR, Kim SH. PPAR Gamma-Mediated G-Protein Coupled Receptor 120 Signaling Pathway Promotes Transcriptional Activation of miR-143 in Adipocytes. Gene (2017) 626:649. doi: 10.1016/j.gene.2017.05.016

34. Kim J-H, Song J, Park KW. The Multifaceted Factor Peroxisome ProliferatorActivated Receptor Gamma (PPAR Gamma) in Metabolism, Immunity, and Cancer. Arch Pharmacal Res (2015) 38:302-12. doi: 10.1007/s12272-0150559-x

35. Yang X, Wang L, Farrar W. A Role for PPAR Gamma in the Regulation of Cytokines in Immune Cells and Cancer. Ppar Res (2008) 2008:961753. doi: $10.1155 / 2008 / 961753$

36. Varga T, Mounier R, Patsalos A, Gogolak P, Peloquin M, Horvath A, et al. Macrophage PPAR Gamma, a Lipid Activated Transcription Factor Controls the Growth Factor GDF3 and Skeletal Muscle Regeneration. Immunity (2016) 45:1038-51. doi: 10.1016/j.immuni.2016.10.016

37. Mounier R, Chazaud B. The PPAR-GDF3 Axis in Macrophages Controls Myogenic Fusion During Muscle Regeneration. M S-Med Sci (2017) 33:46669. doi: $10.1051 / \mathrm{medsci} / 20173305003$

38. Zhu Q, Li C, Yu Z-X, Zou P-F, Meng Q-X, Yao C-L. Molecular and Immune Response Characterizations of IL-6 in Large Yellow Croaker (Larimichthys Crocea). Fish Shellfish Immunol (2016) 50:263-73. doi: 10.1016/ j.fsi.2016.01.041

39. Vansnick J. Interleukin-6 - An Overview. Annu Rev Immunol (1990) 8:25378. doi: 10.1146/annurev.immunol.8.1.253

40. Vonk AG, Netea MG, Van Krieken JH, Iwakura Y, van der Meer JWM, Kullberg BJ. Endogenous Interleukin (IL)-1 Alpha and IL-1 Beta Are Crucial for Host Defense Against Disseminated Candidiasis. J Infect Dis (2006) 193:1419-26. doi: 10.1086/503363

41. Li Q, Wu M, Cui K, Zhu S, Mai K, Ai Q. Characterization of Antiviral Immune Response Induced by Poly(I:C) in Macrophages of Farmed Large Yellow Croaker (Larimichthys Crocea). Fish Shellfish Immunol (2020) 104:663-72. doi: 10.1016/j.fsi.2020.05.066

Conflict of Interest: The authors declare that the research was conducted in the absence of any commercial or financial relationships that could be construed as a potential conflict of interest.

Copyright (C) $2021 \mathrm{Wu}, \mathrm{Li}, \mathrm{Mai}$ and Ai. This is an open-access article distributed under the terms of the Creative Commons Attribution License (CC BY). The use, distribution or reproduction in other forums is permitted, provided the original author(s) and the copyright owner(s) are credited and that the original publication in this journal is cited, in accordance with accepted academic practice. No use, distribution or reproduction is permitted which does not comply with these terms. 